\title{
SU(2)-path integral investigation of Holstein dimer
}

\author{
T. Hakioğlu ${ }^{\mathrm{a}}$, V.A. Ivanov ${ }^{\mathrm{b}, \mathrm{c}}$, M.Ye. Zhuravlev ${ }^{\mathrm{c}, \mathrm{d}, *}$ \\ ${ }^{a}$ Bilkent University, Department of Physics, 06533 Ankara, Turkey \\ ${ }^{\mathrm{b}}$ Laboratorium voor Vaste-Stoffysica en Magnetisme, Katholieke Universiteit Leuven, \\ Celestijnenlaan 200D, 3001, Leuven, Belgium \\ ${ }^{\mathrm{d}}$ Fakultät für Physik, Universität Bielefeld, 33501 Bielefeld 1, Germany \\ ${ }^{\mathrm{c}}$ N.S. Kurnakov Institute of General and Inorganic Chemistry of the RAS, 117907 Moscow, Russia
}

Received 14 September 1999

\begin{abstract}
The SU(2) coherent state path integral is used to investigate the partition function of the Holstein dimer. This approach naturally takes into account the symmetry of the model. The ground-state energy and the number of the phonons are calculated as functions of the parameters of the Hamiltonian. The renormalizations of the phonon frequency and electron orbital energies are considered. The destruction of quasiclassical mean-field solution is discussed. (c) 2000 Elsevier Science B.V. All rights reserved.
\end{abstract}

PACS: 71.38.+i; 63.22.+m; 03.65.DB

Keywords: Holstein dimer; Path integral; Mean-field solution

\section{Introduction}

Despite their simplicity, the dimer models are subject to intense work because of the fact that they represent simple interacting electron-phonon systems of which understanding can provide information about the polaron dynamics for more realistic but equally unsolvable systems. There are at least two reasons for such interest in the dimer models. First of all, we can understand the essential features of the interaction between the electron and phonon degrees of freedom. The other reason is that the dimer models are physical approximations to many organic compounds at the molecular level.

\footnotetext{
* Corresponding author.

E-mail address: zhur@physik.uni-bielefeld.de (M.Ye. Zhuravlev)
} 
There are various analytical (semi-analytical) approaches to the electron-phonon systems in general $[1,2]$ and in particular to the problem of a dimer with a few number of electrons interacting with local vibrations [3,4]. Numerical solution of the two-site polaron problem was performed in Refs. [1,5]. The formation of small polaron was investigated and comparison with analytical results was fulfilled.

The simplest analytical dimer model contains only a single electron interacting with the molecular phonons at two sites. One of the semi-analytical approaches is based on the symmetry of Shrödinger equation and consists in diagonalization of the Shrödinger equation in the electron subspace [6] followed by the analysis (usually numerical) of the obtained equations in the phonon variables [3]. In Ref. [7] the partition function of the dimer Hamiltonian was calculated approximately via path integral using FultonGouterman (FG) transformation [6,8]. In Ref. [9] the equation of motion method was applied to analyze the infrared spectra of a system of noninteracting Holstein dimers.

In the present work, we examine a different method. We apply the SU(2) coherent state path integral to calculate analytically the partition function of the Holstein dimer with a single electron. The advantage of this particular method is that it naturally incorporates the symmetry of the underlying Hamiltonian. We discuss the validity of the obtained results and compare them with those obtained from other analytical approaches.

\section{SU(2) coherent state path integral representation of the partition function of the Holstein dimer}

We start with the one-mode Hamiltonian

$$
\begin{aligned}
H_{\mathrm{dim}}= & -t\left(c_{1}^{+} c_{2}+c_{2}^{+} c_{1}\right)+\omega\left(a_{1}^{+} a_{1}+a_{2}^{+} a_{2}\right) \\
& +g\left(a_{1}^{+}+a_{1}\right) n_{1}+g\left(a_{2}^{+}+a_{2}\right) n_{2}+\varepsilon\left(n_{1}-n_{2}\right)
\end{aligned}
$$

with the one-electron constraint

$$
n_{1}+n_{2}=1 \text {, }
$$

where in the case of no spin-dependent interaction we drop the spin indices from the fermion operators. We can separate in Eq. (1) the one-phonon degree of freedom by rotating the initial phonon coordinates as

$$
a_{1}=\frac{w+v}{\sqrt{2}}, \quad a_{2}=\frac{-w+v}{\sqrt{2}} .
$$

The initial Hamiltonian is then written in a separable form as

$$
H_{\text {dim }}=H_{v}+H_{w},
$$

where

$$
H_{v}=\omega v^{+} v+\frac{g}{\sqrt{2}}\left(v^{+}+v\right)
$$




$$
H_{w}=-t\left(c_{1}^{+} c_{2}+c_{2}^{+} c_{1}\right)+\omega w^{+} w+\left[\frac{g}{\sqrt{2}}\left(w^{+}+w\right)+\varepsilon\right]\left(n_{1}-n_{2}\right) .
$$

Since $v$ and $w$ are separate independent variables, the partition function of the Hamiltonian (1) is the product of the partition function $Z_{v}$ of the displaced Harmonic oscillator (3)

$$
Z_{v}=\frac{\mathrm{e}^{\beta g^{2} / 2 \omega}}{1-\mathrm{e}^{-\beta \omega}}
$$

and the partition function $Z_{w}$ of the Hamiltonian (4). On the other hand, the partition function $Z_{w}$ cannot be calculated exactly. Due to the constraint (2) the Hamiltonian (4) can be naturally rewritten by using the electron pseudo-spin operators in the representation

$$
\begin{aligned}
& \hat{J}_{+}=c_{1}^{+} c_{2}, \quad \hat{J}_{-}=c_{2}^{+} c_{1}, \quad \hat{J}_{0}=\frac{1}{2}\left(c_{1}^{+} c_{1}-c_{2}^{+} c_{2}\right), \\
& H_{w}=-t\left(\hat{J}_{+}+\hat{J}_{-}\right)+\left[\frac{g}{\sqrt{2}}\left(w^{+}+w\right)+\varepsilon\right] 2 \hat{J}_{0}+\omega w^{+} w .
\end{aligned}
$$

Now, it is more convenient for further calculation to apply the rotation in the spin space about the $x$-axis by $\pi / 2$ :

$$
\hat{J}_{y} \rightarrow \hat{J}_{0}, \quad \hat{J}_{0} \rightarrow-\hat{J}_{y},
$$

where

$$
J_{x}=\frac{J_{+}+J_{-}}{2}, \quad J_{y}=\frac{J_{+}-J_{-}}{2 i}, \quad\left[J_{x}, J_{y}\right]=\mathrm{i} J_{0} .
$$

We then obtain the following form of the spin Hamiltonian:

$$
\begin{aligned}
H_{w}= & \omega w^{+} w-\left[t-\mathrm{i}\left(\varepsilon+\frac{g}{\sqrt{2}}\left(w^{+}+w\right)\right)\right] \hat{J}_{+} \\
& -\left[t+\mathrm{i}\left(\varepsilon+\frac{g}{\sqrt{2}}\left(w^{+}+w\right)\right)\right] \hat{J}_{-} .
\end{aligned}
$$

The partition function of the Hamiltonian (6) can be represented as a path integral over SU(2) variables [10,11] and phonon variables:

$$
\begin{aligned}
Z_{w}= & \operatorname{Tr} \mathrm{e}^{-\beta H_{w}}=\int \mathrm{D} w \mathrm{D} \bar{w} \mathrm{D} \mu_{\mathrm{SU}(2)} \exp \left\{\int_{0}^{\beta}[-\bar{w} \dot{w}-\omega \bar{w} w\right. \\
& +\frac{1}{2} \frac{\bar{\alpha}(\dot{\tau}) \alpha(\tau)-\bar{\alpha}(\tau) \dot{\alpha}(\tau)}{1+|\alpha|^{2}}+\frac{1}{2}\left[t+\mathrm{i}\left(\varepsilon+\frac{g}{\sqrt{2}}(\bar{w}(\tau)+w(\tau))\right)\right] \frac{\alpha(\tau)}{1+|\alpha|^{2}} \\
& \left.\left.+\frac{1}{2}\left[t-\mathrm{i}\left(\varepsilon+\frac{g}{\sqrt{2}}(\bar{w}(\tau)+w(\tau))\right)\right] \frac{\bar{\alpha}(\tau)}{1+|\alpha|^{2}}\right] \mathrm{~d} \tau\right\}
\end{aligned}
$$

where $\mathrm{SU}(2)$ invariant measure

$$
D \mu_{\mathrm{SU}(2)}=\prod_{\tau} \frac{2}{\pi} \frac{d^{2} \alpha(\tau)}{\left(1+|\alpha(\tau)|^{2}\right)^{2}} .
$$

For the simplicity of the numerical calculations at the last stage we use in the present consideration the particular representation with the eigenvalue $J=\frac{1}{2}$ for the $\mathrm{SU}(2)$ 
path integral. Just the same the analytical calculations can be performed for arbitrary representation index. The evaluation of the partiton function (7) will be performed in two steps. The Hamiltonian $H_{w}$ is linear in terms of the SU(2) generators and the path integral over the $\mathrm{SU}(2)$ variables can be calculated by the method developed in Ref. [12]. The resulting path integral over the phonon variables $w(\tau), \bar{w}(\tau)$ will be calculated in the stationary phase approximation.

According to the approach [12] the path integral (7) can be calculated by the change of integration variables $\alpha(\tau), \bar{\alpha}(\tau)$. The result is expressed in terms of the auxiliary functions $z(\tau), \bar{z}(\tau)$ which define the change of variables. The details of the methods can be found in Refs. [12,13]. Therefore, the integration over the $\mathrm{SU}(2)$ variables yields

$$
Z_{w}=\int D \mu_{\mathrm{SU}(2)} \exp \left\{\int_{0}^{\beta}[-\bar{w} \dot{w}-\omega \bar{w} w] \mathrm{d} \tau+\log \frac{\sinh \int_{0}^{\beta} \Omega(\tau) \mathrm{d} \tau}{\sinh (1 / 2) \int_{0}^{\beta} \Omega(\tau) \mathrm{d} \tau}\right\}
$$

where

$$
\begin{aligned}
\Omega(\tau)= & {\left[t+\mathrm{i}\left(\varepsilon+\frac{g}{\sqrt{2}}(\bar{w}(\tau)+w(\tau))\right)\right] z(\tau) } \\
& -\left[t-\mathrm{i}\left(\varepsilon+\frac{g}{\sqrt{2}}(\bar{w}(\tau)+w(\tau))\right)\right] \bar{z}(\tau) .
\end{aligned}
$$

The auxiliary functions $z(\tau), \bar{z}(\tau)$ are defined through the following system of equations:

$$
\begin{aligned}
\frac{\mathrm{d}}{\mathrm{d} \tau} z(\tau)+ & {\left[t+\mathrm{i}\left(\varepsilon+\frac{g}{\sqrt{2}}(\bar{w}(\tau)+w(\tau))\right)\right] z^{2}(\tau) } \\
- & {\left[t-\mathrm{i}\left(\varepsilon+\frac{g}{\sqrt{2}}(\bar{w}(\tau)+w(\tau))\right)\right]=0 } \\
\frac{\mathrm{d}}{\mathrm{d} \tau} \bar{z}(\tau)- & {\left[t-\mathrm{i}\left(\varepsilon+\frac{g}{\sqrt{2}}(\bar{w}(\tau)+w(\tau))\right)\right] \bar{z}^{2}(\tau) } \\
+ & {\left[t+\mathrm{i}\left(\varepsilon+\frac{g}{\sqrt{2}}(\bar{w}(\tau)+w(\tau))\right)\right]=0 }
\end{aligned}
$$

with the usual periodic boundary conditions $z(0)=z(\beta), \bar{z}(0)=\bar{z}(\beta)$.

Simplifying the term with the logarithm in Eq. (9) we obtain the following expression for the partition function:

$$
\begin{aligned}
Z_{w}= & Z_{w-}+Z_{w+}=\int \mathrm{D} w \operatorname{D} \bar{w} \exp \left(S_{-}\right)+\int \mathrm{D} w \operatorname{D} \bar{w} \exp \left(S_{+}\right) \\
= & \int \mathrm{D} w \mathrm{D} \bar{w} \exp \int_{0}^{\beta}\{-\bar{w} \dot{w}-\omega \bar{w} w-\Omega / 2\} \mathrm{d} \tau \\
& +\int \mathrm{D} w \mathrm{D} \bar{w} \exp \int_{0}^{\beta}\{-\bar{w} \dot{w}-\omega \bar{w} w+\Omega / 2\} \mathrm{d} \tau
\end{aligned}
$$




$$
\begin{aligned}
= & \int \mathrm{D} w \mathrm{D} \bar{w} \exp \int_{0}^{\beta}\{-\bar{w} \dot{w}-\omega \bar{w} w \\
& \left.+\frac{1}{2} t(\bar{z}+z)+\frac{1}{2} \mathrm{i}\left(\varepsilon+\frac{g}{\sqrt{2}}(\bar{w}+w)\right)(z-\bar{z})\right\} \mathrm{d} \tau \\
& +\int \mathrm{D} w \mathrm{D} \bar{w} \exp \int_{0}^{\beta}\left\{-\bar{w} \dot{w}-\omega \bar{w} w-\frac{1}{2} t(\bar{z}+z)\right. \\
& \left.-\frac{1}{2} \mathrm{i}\left(\varepsilon+\frac{g}{\sqrt{2}}(\bar{w}+w)\right)(z-\bar{z})\right\} \mathrm{d} \tau .
\end{aligned}
$$

It is interesting to note that the representation of the dimer partition function as a sum

$$
Z=Z_{+}+Z_{-}
$$

can be obtained [7] by applying the Fulton-Gouterman transformation [6,8] to the initial Hamiltonian. It should be noted that the "functional" Fulton-Gouterman-like representation (12) can still be obtained by using method [12] even for those Hamiltonians for which the usual FG transformation is inapplicable.

Eq. (11) form a set of coupled Riccati equations which cannot be solved exactly as a functional of the arbitrary functions $\bar{w}(\tau), w(\tau)$. In addition to this fact, the system in Eq. (12) fall outside the class of a few exactly calculable non-Gaussian path integrals. We present the evaluation of the path integral (12) in detail in the stationary phase approximation for $Z_{w-\text {. }}$

\section{Stationary phase approximation}

As the first step, we replace the trajectories $\bar{w}(\tau), w(\tau)$ by their stationary values obtained from the stationary phase conditions $\delta S_{-} / \delta w(\tau)=0, \delta S_{-} / \delta \bar{w}(\tau)=0$ as

$$
\begin{aligned}
\frac{\delta S_{-}}{\delta \bar{w}(\tau)}= & \left(-\frac{\mathrm{d}}{\mathrm{d} \tau}-\omega\right) w(\tau)+\frac{\mathrm{i} g}{2 \sqrt{2}}(z(\tau)-\bar{z}(\tau))+\frac{1}{2} \int_{0}^{\beta}\left\{t\left(\frac{\delta z\left(\tau^{\prime}\right)}{\delta \bar{w}(\tau)}+\frac{\delta \bar{z}\left(\tau^{\prime}\right)}{\delta \bar{w}(\tau)}\right)\right. \\
& \left.+\mathrm{i}\left(\varepsilon+\frac{g}{\sqrt{2}}(\bar{w}+w)\right)\left(\frac{\delta z\left(\tau^{\prime}\right)}{\delta \bar{w}(\tau)}-\frac{\delta \bar{z}\left(\tau^{\prime}\right)}{\delta \bar{w}(\tau)}\right)\right\} \mathrm{d} \tau^{\prime}=0
\end{aligned}
$$

and

$$
\begin{aligned}
\frac{\delta S_{-}}{\delta w(\tau)}= & \left(\frac{\mathrm{d}}{\mathrm{d} \tau}-\omega\right) \bar{w}(\tau)+\frac{\mathrm{i} g}{2 \sqrt{2}}(z(\tau)-\bar{z}(\tau))+\frac{1}{2} \int_{0}^{\beta}\left\{t\left(\frac{\delta z\left(\tau^{\prime}\right)}{\delta w(\tau)}+\frac{\delta \bar{z}\left(\tau^{\prime}\right)}{\delta w(\tau)}\right)\right. \\
& \left.+\mathrm{i}\left(\varepsilon+\frac{g}{\sqrt{2}}(\bar{w}+w)\right)\left(\frac{\delta z\left(\tau^{\prime}\right)}{\delta w(\tau)}-\frac{\delta \bar{z}\left(\tau^{\prime}\right)}{\delta w(\tau)}\right)\right\} \mathrm{d} \tau^{\prime}=0
\end{aligned}
$$

with periodical boundary conditions $\bar{w}(0)=\bar{w}(\beta), w(0)=w(\beta)$. In accordance with the general scheme of stationary phase approximation [14], the partition function $Z_{w-}$ is represented as

$$
\int \mathrm{D} \bar{w} \mathrm{D} w \mathrm{e}^{S_{-}} \sim \mathrm{e}^{S_{0-}} \int \mathrm{D} \bar{w} \mathrm{D} w \mathrm{e}^{\delta^{2} S_{-}}=\mathrm{e}^{S_{0-}}(\operatorname{Det} L)^{-1},
$$


where we denote by $S_{0-}$ the zeroth-order term (the stationary solution) for the action $S_{-}$. Here $L$ is the kernel of the non-linear integral operator defined by the second variation of the action $S_{-}$. Eqs. (14) and (15) contain the variational derivatives of the auxiliary functions $z(\tau), \bar{z}(\tau)$. The equations for $\delta z^{*}\left(\tau^{\prime}\right) / \delta w^{* *}(\tau)$ can be obtained by variation of Eq. (11) as

$$
\begin{aligned}
& \frac{\mathrm{d}}{\mathrm{d} \tau^{\prime}} \frac{\delta z\left(\tau^{\prime}\right)}{\delta w^{*}(\tau)}+2\left[t+\mathrm{i}\left(\varepsilon+\frac{g}{\sqrt{2}}\left(w\left(\tau^{\prime}\right)+\bar{w}\left(\tau^{\prime}\right)\right)\right)\right] z\left(\tau^{\prime}\right) \frac{\delta z\left(\tau^{\prime}\right)}{\delta w^{*}(\tau)} \\
&+ \mathrm{i} \frac{g}{\sqrt{2}} \delta\left(\tau^{\prime}-\tau\right) z^{2}\left(\tau^{\prime}\right)+\mathrm{i} \frac{g}{\sqrt{2}} \delta\left(\tau^{\prime}-\tau\right)=0, \\
& \frac{\mathrm{d}}{\mathrm{d} \tau^{\prime}} \frac{\delta \bar{z}\left(\tau^{\prime}\right)}{\delta w^{*}(\tau)}-2\left[t-\mathrm{i}\left(\varepsilon+\frac{g}{\sqrt{2}}\left(w\left(\tau^{\prime}\right)+\bar{w}\left(\tau^{\prime}\right)\right)\right)\right] \bar{z}\left(\tau^{\prime}\right) \frac{\delta z\left(\tau^{\prime}\right)}{\delta w^{*}(\tau)} \\
&+\mathrm{i} \frac{g}{\sqrt{2}} \delta\left(\tau^{\prime}-\tau\right) \bar{z}^{2}\left(\tau^{\prime}\right)+\mathrm{i} \frac{g}{\sqrt{2}} \delta\left(\tau^{\prime}-\tau\right)=0 .
\end{aligned}
$$

Periodic boundary conditions on variational derivatives imply that

$$
\frac{\delta z^{*}\left(\tau^{\prime}=0\right)}{\delta w^{* *}(\tau)}=\frac{\delta z^{*}\left(\tau^{\prime}=\beta\right)}{\delta w^{* *}(\tau)},
$$

where $z^{*}(\tau)=z(\tau)$ or $\bar{z}(\tau)$ and $w^{* *}(\tau)=w(\tau)$ or $\bar{w}(\tau)$. From here on the notations “*,**" denote the conjugation or the absence of the conjugation, respectively. The second variation of the action $\delta^{2} S_{-}$is expressed in terms of the first and the second variational derivatives of the auxiliary functions $z(\tau), \bar{z}(\tau)$. By variation of Eqs. (17) and (18) we obtain

$$
\begin{aligned}
& \frac{\mathrm{d}}{\mathrm{d} \tau^{\prime}} \frac{\delta^{2} z\left(\tau^{\prime}\right)}{\delta w^{*}(\tau) \delta w^{* *}(\sigma)}+2\left[t+\mathrm{i}\left(\varepsilon+\frac{g}{\sqrt{2}}\left(w\left(\tau^{\prime}\right)+\bar{w}\left(\tau^{\prime}\right)\right)\right)\right] z\left(\tau^{\prime}\right) \frac{\delta^{2} z\left(\tau^{\prime}\right)}{\delta w^{*}(\tau) \delta w^{* *}(\sigma)} \\
& +2\left[t+\mathrm{i}\left(\varepsilon+\frac{g}{\sqrt{2}}\left(w\left(\tau^{\prime}\right)+\bar{w}\left(\tau^{\prime}\right)\right)\right)\right] \frac{\delta z\left(\tau^{\prime}\right)}{\delta w^{*}(\tau)} \frac{\delta z\left(\tau^{\prime}\right)}{\delta w^{* *}(\sigma)} \\
& \quad+2 \mathrm{i} \frac{g}{\sqrt{2}} z\left(\tau^{\prime}\right)\left(\frac{\delta z\left(\tau^{\prime}\right)}{\delta w^{*}(\tau)} \delta\left(\tau^{\prime}-\sigma\right)+\frac{\delta z\left(\tau^{\prime}\right)}{\delta w^{* *}(\sigma)} \delta\left(\tau^{\prime}-\tau\right)\right)=0
\end{aligned}
$$

and

$$
\begin{aligned}
& \frac{\mathrm{d}}{\mathrm{d} \tau^{\prime}} \frac{\delta^{2} \bar{z}\left(\tau^{\prime}\right)}{\delta w^{*}(\tau) \delta w^{* *}(\sigma)}-2\left[t-\mathrm{i}\left(\varepsilon+\frac{g}{\sqrt{2}}\left(w\left(\tau^{\prime}\right)+\bar{w}\left(\tau^{\prime}\right)\right)\right)\right] \bar{z}\left(\tau^{\prime}\right) \frac{\delta^{2} \bar{z}\left(\tau^{\prime}\right)}{\delta w^{*}(\tau) \delta w^{* *}(\sigma)} \\
& \quad-2\left[t-\mathrm{i}\left(\varepsilon+\frac{g}{\sqrt{2}}\left(w\left(\tau^{\prime}\right)+\bar{w}\left(\tau^{\prime}\right)\right)\right)\right] \frac{\delta \bar{z}\left(\tau^{\prime}\right)}{\delta w^{*}(\tau)} \frac{\delta \bar{z}\left(\tau^{\prime}\right)}{\delta w^{* *}(\sigma)} \\
& \quad+2 \mathrm{i} \frac{g}{\sqrt{2}} \bar{z}\left(\tau^{\prime}\right)\left(\frac{\delta \bar{z}\left(\tau^{\prime}\right)}{\delta w^{*}(\tau)} \delta\left(\tau^{\prime}-\sigma\right)+\frac{\delta \bar{z}\left(\tau^{\prime}\right)}{\delta w^{* *}(\sigma)} \delta\left(\tau^{\prime}-\tau\right)\right)=0
\end{aligned}
$$

Hereupon, we replace the functions $z(\tau), \bar{z}(\tau), \bar{w}(\tau)+w(\tau)$ in Eqs. (17) $-(20)$ by the constants $z_{0}, \bar{z}_{0}$ and $W_{0-}$ correspondingly obtained as the time-independent solutions of 
Eqs. (11), (14) and (15). These equations give

$$
\begin{aligned}
& z_{0}=\sqrt{\frac{t-\mathrm{i}\left(\varepsilon+(g / \sqrt{2}) W_{0-}\right)}{t+\mathrm{i}\left(\varepsilon+(g / \sqrt{2}) W_{0-}\right)}} \operatorname{sign}\left(\varepsilon+\frac{g}{\sqrt{2}} W_{0-}\right) \\
& \bar{z}_{0}=\sqrt{\frac{t+\mathrm{i}\left(\varepsilon+(g / \sqrt{2}) W_{0-}\right)}{t-\mathrm{i}\left(\varepsilon+(g / \sqrt{2}) W_{0-}\right)}} \operatorname{sign}\left(\varepsilon+\frac{g}{\sqrt{2}} W_{0-}\right) .
\end{aligned}
$$

We choose the branches of the square roots in Eq. (21) in such a way that the exactly solvable limits $(g=0$ and $t=0)$ can be reproduced. The factors $\operatorname{sign}\left(\varepsilon+(g / \sqrt{2}) W_{0-}\right)$ imply that we should make the branch cut along the positive real axis. Taking the sum of Eqs. (14) and (15) we get

$$
W_{0-}=\frac{\sqrt{2} g\left(\varepsilon+(g / \sqrt{2}) W_{0-}\right)}{\omega E_{0-}} \operatorname{sign}\left(\varepsilon+\frac{g}{\sqrt{2}} W_{0-}\right),
$$

where

$$
E_{0-}=\sqrt{t^{2}+\left(\varepsilon+\frac{g}{\sqrt{2}} W_{0-}\right)^{2}} .
$$

Now, the differential Eqs. (17)-(20) can be solved for the first and second variational derivatives of $z, \bar{z}$ with respect to $w, \bar{w}$. Then, these variational derivatives should be substituted in the second variations of action $S_{0-}$ obtained by variation of Eqs. (14) and (15). As a result the following form of the second variational derivatives of the action can get:

$$
\frac{\delta^{2} S_{-}}{\delta w^{*}(\tau) \delta w^{* *}(\sigma)}=L_{0}+\frac{g^{2} t^{2} \operatorname{sign}\left(\varepsilon+(g / \sqrt{2}) W_{0-}\right)}{2\left(t^{2}+\left(\varepsilon+(g / \sqrt{2}) W_{0-}\right)^{2}\right)} \frac{\cosh 2 E_{0-}(\beta / 2-|\sigma-\tau|)}{\sinh \beta E_{0-}} .
$$

Here $L_{0}$ is the second variation of the harmonic part of the action. In the framework of the stationary phase approximation, the second variation defines the Gaussian path integral with the kernel depending on the difference of the time $\tau-\sigma$. Corresponding path integral is calculated in the Appendix.

Using Eq. (23) we obtain for the partition function

$$
Z_{-} \approx Z_{v} \cdot Z_{w-}=\frac{\mathrm{e}^{-\beta E_{b}}}{\left(1-\mathrm{e}^{-\beta \omega}\right)\left(1-\mathrm{e}^{-\beta \omega_{1-}}\right)} \frac{1-\mathrm{e}^{-2 \beta E_{0-}}}{1-\mathrm{e}^{-\beta \omega_{2-}}} .
$$

Here

$$
\begin{aligned}
& E_{b}=\frac{\omega W_{0-}^{2}}{4}-2 E_{0-}+\frac{\omega_{1-}+\omega_{2-}-\omega}{2}-\frac{g^{2}}{2 \omega} \\
& \omega_{1-, 2-}^{2}=\frac{\left(4 E_{0-}^{2}+\omega^{2}\right) \pm \sqrt{\left(4 E_{0-}^{2}-\omega^{2}\right)^{2}+\left(16 g^{2} t^{2} \omega / E_{0-}\right)}}{2} .
\end{aligned}
$$


The other partition function $Z_{+}=Z_{v} Z_{w+}$ can be calculated separately in a similar way which yields

$$
Z_{+}=\frac{\mathrm{e}^{-\beta E_{a}}}{\left(1-\mathrm{e}^{-\beta \omega}\right)\left(1-\mathrm{e}^{-\beta \omega_{1+}}\right)} \frac{1-\mathrm{e}^{-2 \beta E_{0+}}}{1-\mathrm{e}^{-\beta \omega_{2+}}},
$$

with

$$
\begin{aligned}
& E_{a}=\frac{\omega W_{0+}^{2}}{4}+E_{0+}\left(\operatorname{sign}\left(\varepsilon+\frac{g}{\sqrt{2}} W_{0+}\right)-1\right)+\frac{\omega_{1+}+\omega_{2+}-\omega}{2}-\frac{g^{2}}{2 \omega} \\
& \omega_{1+, 2+}^{2}=\frac{\left(4 E_{0}^{2}+\omega^{2}\right) \pm \sqrt{\left(4 E_{0+}^{2}-\omega^{2}\right)^{2}-\left(16 g^{2} t^{2} \omega / E_{0+}\right)}}{2} \\
& E_{0+}=\sqrt{t^{2}+\left(\varepsilon+(g / \sqrt{2}) W_{0+}\right)^{2}} .
\end{aligned}
$$

The condition for $W_{0+}$ is given by

$$
W_{0+}=-\frac{\sqrt{2} g\left(\varepsilon+(g / \sqrt{2}) W_{0+}\right)}{\omega E_{0}} \operatorname{sign}\left(\varepsilon+\frac{g}{\sqrt{2}} W_{0+}\right) .
$$

To find the ground-state energy of the dimer one should represent the partition function of the system in the form $Z=\sum_{n} \exp \left(-\beta E_{n}\right)$. The least energy among the $E_{n}$ represents the ground-state energy. Expanding the partition functions (24) and (26) we see that the ground-state energy is the least energy between $E_{a}$ and $E_{b}$. We will see when analyzing the limiting cases $g=0$ that $Z_{-}$originates from the binding state of the electron, so $E_{b}(g=0)<E_{a}(g=0)$. The same inequality holds for $t=0$ limit. This interrelation is true for $g, t \neq 0$ too. So, $E_{b}$ represents the ground-state energy of the dimer.

\section{Exactly solvable limits}

In this section, we investigate the exactly solvable limits $g=0$ and $t=0$.

(a) The non-interacting limit: $g=0$

The electron and phonon subsystems are obviously decoupled in this case and the partition function of the Hamiltonian (4) takes the form of a product of the electron and phonon parts

$$
\left.Z_{w}\right|_{g=0}=Z_{\mathrm{ph}} Z_{\mathrm{el}} .
$$

The phonon partition function now is that of the harmonic oscillator, $Z_{\mathrm{ph}}=(1-$ $\exp (-\beta \omega))^{-1}$. Electron part of Hamiltonian can be easily diagonalized in terms of binding and anti-binding orbitals with the eigen energies

$$
\lambda_{b}=-\sqrt{\varepsilon^{2}+t^{2}}, \quad \lambda_{a}=\sqrt{\varepsilon^{2}+t^{2}} .
$$


Therefore, the partition function (29) acquires the following form:

$$
\left.Z_{w}\right|_{g=0}=\frac{\mathrm{e}^{-\beta \sqrt{\varepsilon^{2}+t^{2}}}}{1-\mathrm{e}^{-\beta \omega}}+\frac{\mathrm{e}^{\beta \sqrt{\varepsilon^{2}+t^{2}}}}{1-\mathrm{e}^{-\beta \omega}} .
$$

One can easily find that we obtain in the limit being considered $W_{0+}=W_{0-}=0$. Hence

$$
\omega_{1 \pm, 2 \pm}=\left[\begin{array}{l}
\omega^{2} \\
4 E_{0}^{2}
\end{array}\right] \text {. }
$$

Therefore, in the non-interacting limit, the sum of the expressions (24) and (26) is transformed into Eq. (30). This implies that the partiton functions (24) and (26) correspond to symmetric (binding) and antisymmetric (antibinding) electronic states. In the work [7] the representation of the partition function of a dimer as $Z=Z_{+}+Z_{-}$ was obtained. It is interesting to note that the summands $Z_{+}, Z_{-}$in that work differ from the summands of the same names which are obtained in the present work after integrating (7) over $\mathrm{SU}(2)$ variables. The exact calculation of the path integral with non-linear action corresponding to this solvable limit is represented in Ref. [15].

(b) The limit $t=0$.

Now we turn to the other solvable case, $t=0$. In this limit, the electron energies of the Hamiltonian (4) are given by $\lambda_{1}=\varepsilon$ or $\lambda_{2}=-\varepsilon$ depending on the site occupied (unoccupied) by the electron. In both cases, the phonon partition function of Hamiltonian (4) coincides with $Z_{v}$, Eq. (5), so that we have

$$
\left.Z_{w}\right|_{t=0}=\frac{\exp \left(\beta g^{2} / 2 \omega\right)(\exp (\beta \varepsilon)+\exp (-\beta \varepsilon))}{1-\exp (-\beta \omega)} .
$$

In the case, $t=0$ under consideration here it is easy to verify that $W_{0-}=-W_{0+}=\sqrt{2 g} / \omega$ and for $\omega_{1,2}^{ \pm}$the condition (31) is still valid. As a result, the partition function $Z_{w_{-}}$in Eq. (24) is transformed into the first term in Eq. (32) whereas, $Z_{+}$, (26) is transformed into the second one. Therefore, our general expression reproduces both the exactly solvable limiting cases.

Lastly, we would like to consider the dependence of exponents in Eqs. (24) and (26) on $\varepsilon$. We notice from Eqs. (22) and (28) that $W_{0+}=W_{0-}=0$ for $\varepsilon=0$ and $g^{2} / \omega t<1$. Nevertheless, the complicated renormalization (25), (27) of the binding and antibinding energies takes place. In the general case, $\varepsilon \neq 0$ it was found in Ref. [9] that the additional distortion of the dimer takes place resulting in the renormalization of the binding orbital energy in adiabatic approximation $-\sqrt{t^{2}+\varepsilon^{2}} \rightarrow-\sqrt{t^{2}+(\varepsilon+\Delta \varepsilon)^{2}}$. In our approach the additional renormalizations differ for $Z_{-}$and $Z_{+}$and they are represented by the general expressions (25) and (27).

\section{Discussion and conclusion}

Due to the stationary phase approximation we obtain a new effective constant of expansion $g^{\text {eff }}=g^{2} t^{2} / \omega E_{0}^{3}$ which differs from the initial electron-phonon coupling 


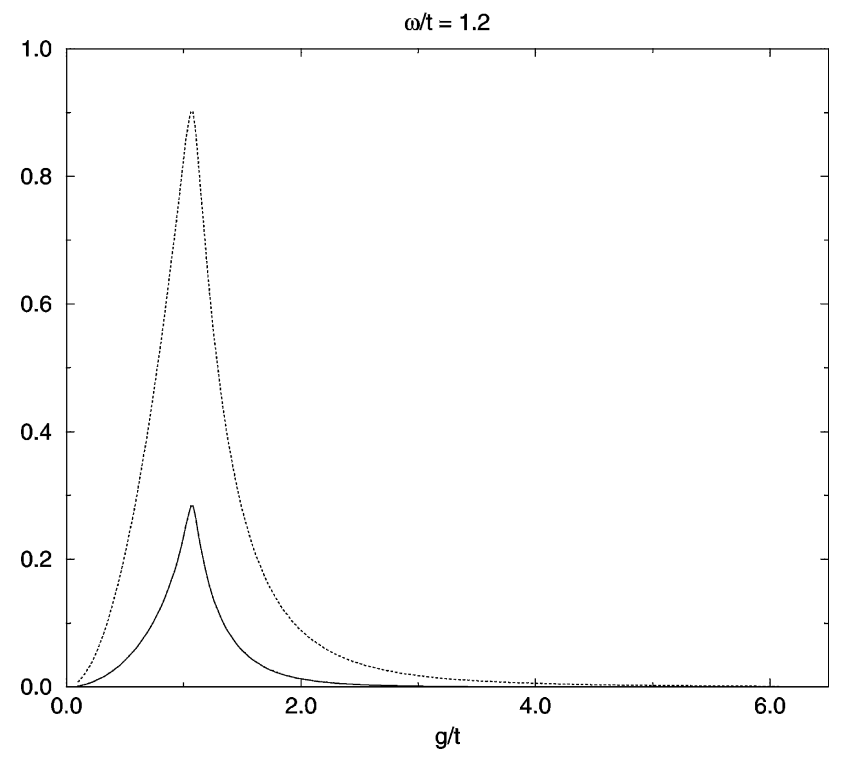

Fig. 1. The effective coupling constant $g^{\text {eff }}$ as a function of $g$ for $\omega / t=1.2$ (solid line) and the ratio $E_{b 1} / E_{b 0}$ (dotted line).

constant $g / \omega$. In this context, we refer to our approach as "non-perturbative". This new constant can still be made small for sufficiently large $g / \omega$. The validity of the approximation can be estimated by the comparison of the contribution into the ground-state energy from the stationary trajectories, $E_{b 0}=\left.S_{-}\right|_{0}$ (Eq. (A.1)) and $\delta^{2} S_{-}$(quantum fluctuations $)$. The contribution from the last term equals $E_{b 1}=\left[\left(\omega_{1-}+\omega_{2-}-\omega\right) / 2\right]-E_{0}$. We cannot define rigorously the region of validity of the stationary phase approximation. The general opinion is that the approximation is adequate if the contribution from the stationary trajectories is dominant. The ratio $E_{b 1} / E_{b 0}$ is represented by Figs. 1 and 2. It shows that our approximation is adequate in the whole range of the parameters except a narrow interval. The other criteria is the ratio of the effective expansion constant to the phonon frequency $g^{2} t^{2} / E_{0}^{3} \omega$. This ratio is represented by Figs. 1 and 2 as well.

Let us consider the ground-state energy $E_{b}$ for various relationships between the parameters of the model. For $\varepsilon=0$ we can obtain an explicit expression for $E_{b}$. For weak electron-phonon interaction $g^{2} / \omega t<1$ we get $W_{0-}=0$. Hence,

$$
\begin{aligned}
E_{b}= & -2 t-\frac{g^{2}}{2 \omega}-\frac{\omega}{2}+\frac{1}{2} \sqrt{\omega^{2}+4 t^{2}+\sqrt{\left[\omega^{2}-4 t^{2}\right]^{2}+16 g^{2} t^{2}}} \\
& +\frac{1}{2} \sqrt{\omega^{2}+4 t^{2}-\sqrt{\left[\omega^{2}-4 t^{2}\right]^{2}+16 g^{2} t^{2}}} .
\end{aligned}
$$




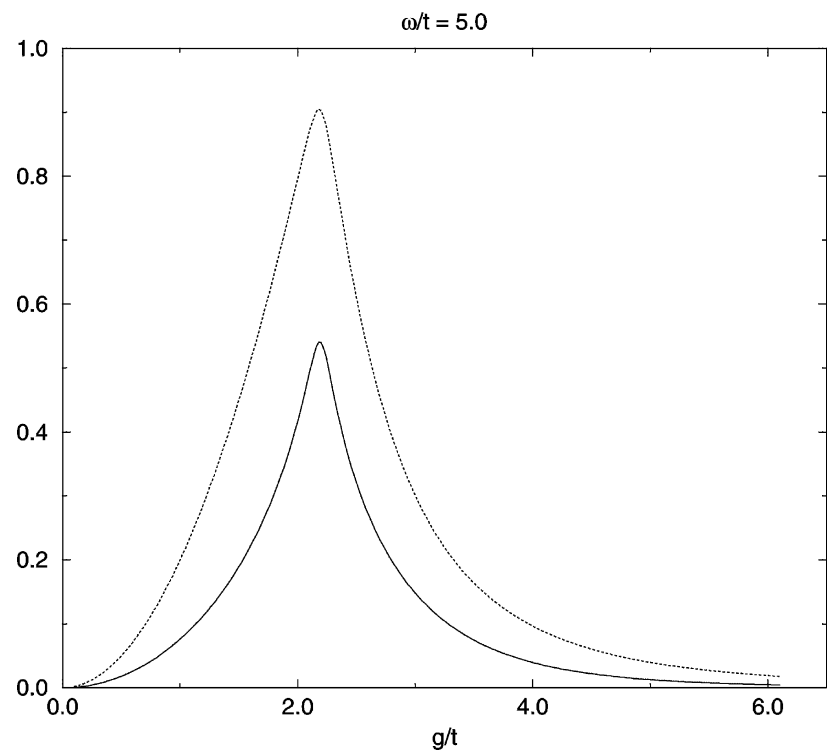

Fig. 2. The effective coupling constant $g^{\text {eff }}$ as a function of $g$ for $\omega / t=5.0$ (solid line) and the ratio $E_{b 1} / E_{b 0}$ (dotted line).

We can expand the square roots for some typical relative strengths of $\omega, t, g$. We have for instance

$$
\text { if } g \ll \omega \ll t, \quad \text { then } E_{b} \simeq-t-\frac{g^{2}}{2 \omega}-\frac{g^{2}}{4 t}+\frac{g^{2} \omega}{8 t^{2}},
$$

and

$$
\text { if } g \ll t \ll \omega, \quad \text { then } E_{b} \simeq-t+\frac{g^{2} t}{2 \omega^{2}}-\frac{g^{2}}{\omega} .
$$

In the case, $g^{2} / \omega t>1$ we find that

$$
W_{0-}=\frac{\sqrt{2}}{g} \sqrt{\frac{g^{4}}{\omega^{2}}-t^{2}},
$$

and

$$
E_{b} \simeq-\frac{g^{2}}{\omega}-\frac{t^{2} \omega}{2 g^{2}}-\frac{t^{2} \omega^{3}}{4 g^{4}} .
$$

The first terms of ground-state energy in Eq. (35) coincides with the expression obtained in Ref. [16] for the lattice in the strongly interacting limit.

Another quantity which is often calculated is the renormalized phonon frequency. Our analysis indicates that a realistic picture is more involved than a simple calculation of the renormalization of the initial phonon frequency. The obtained structure of the dimer partition function can be treated as an effective splitting of the original phonon frequency $\omega$. A similar splitting was obtained in Ref. [7] in the framework of flat 


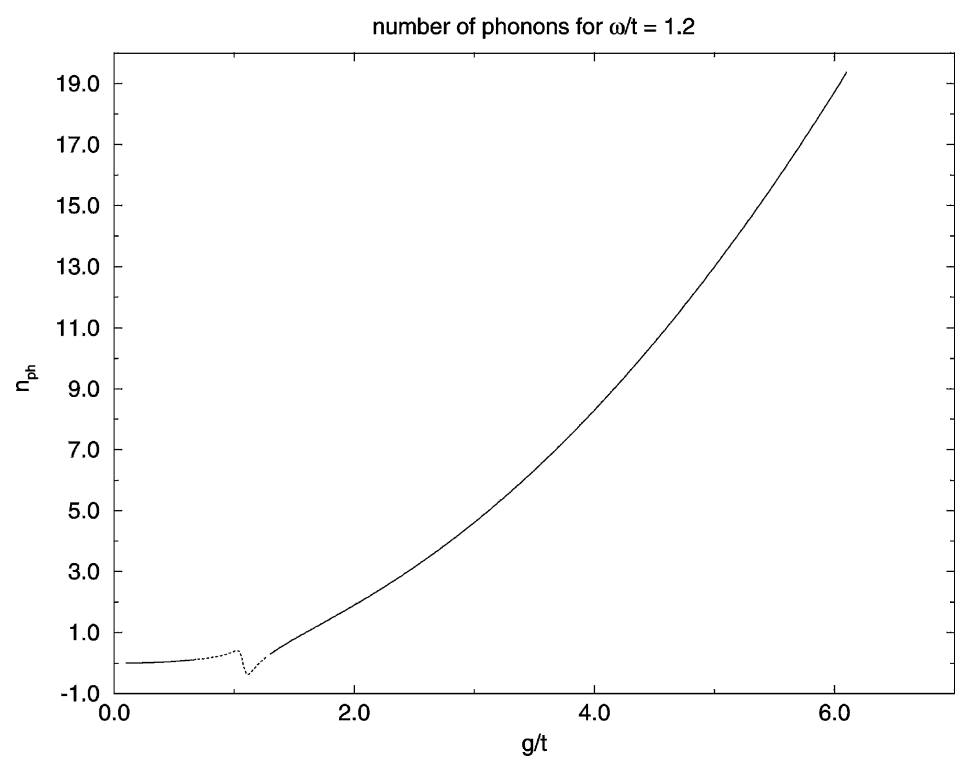

Fig. 3. The number of phonons as a function of $g$ for $\omega / t=1.2$. The non-valid part of the curve is marked as a dotted line.

coherent state path integral approach. However, strongly nonlinear action hampered the applicability of the stationary phase approximation in the flat path integral in Ref. [7].

Another physical quantity which can be calculated by the obtained partition function is the thermal phonon occupation factors,

$$
n_{\mathrm{ph}}=-\frac{1}{Z \beta} \frac{\mathrm{d} Z}{\mathrm{~d} \omega},
$$

which are presented in Figs. 3-4 for such low temperatures $(\beta t=10000)$ that the dominant contribution from the ground-state energy $E_{b}$ is sufficient to consider. In the narrow interval of the electron-phonon coupling constant $g$, where our approach is inapplicable the number of the phonons calculated in accordance with Eq. (35) becomes negative. We suppose that the smooth transition to small polaron picture takes place in this area.

The critical value of the ratio $g / t$, where derivative $\partial n_{\mathrm{ph}} / \partial g$ becomes negative is calculated as a function of phonon frequency $\omega / t$ (Fig. 5). The obtained dependence is linear except in the region of small $g$. It corresponds to "self-trapping line" of the Ref. [17].

Accordingly to Ref. [18] the non-trivial part of the problem of one electron in Holstein chain is the same as in two-site problem. So, our consideration concerning the mean-field description of single-electron coupling with phonons and critical value of $\omega$ as a function of the coupling constant $g$ is also valid for the chain. 


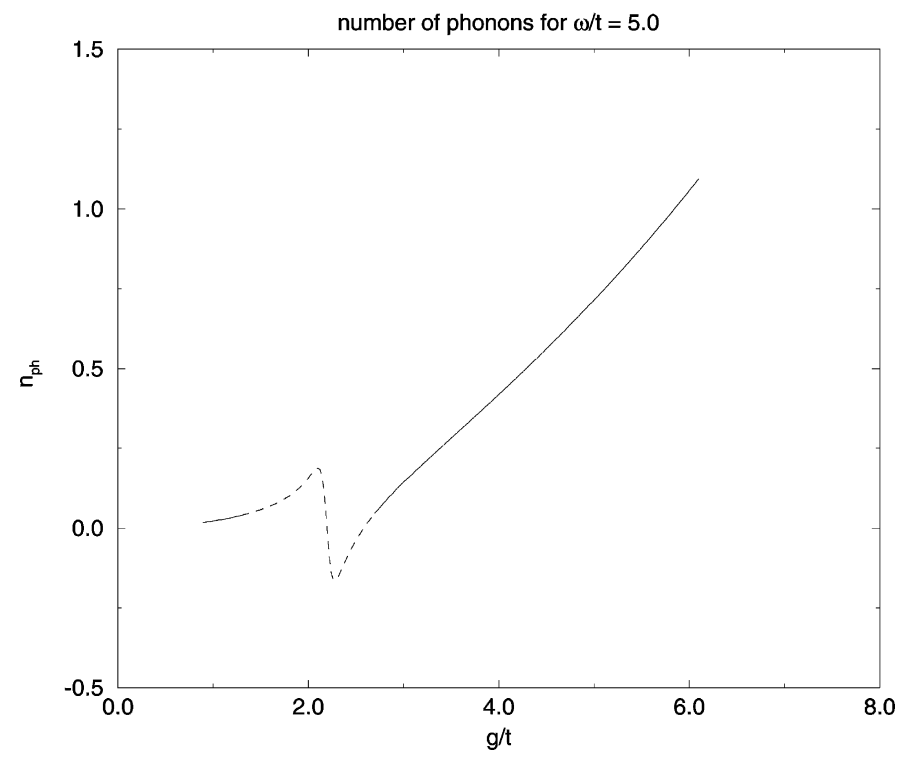

Fig. 4. The number of phonons as a function of $g$ for $\omega / t=5.0$. The non-valid part of the curve is marked as a dashed line.

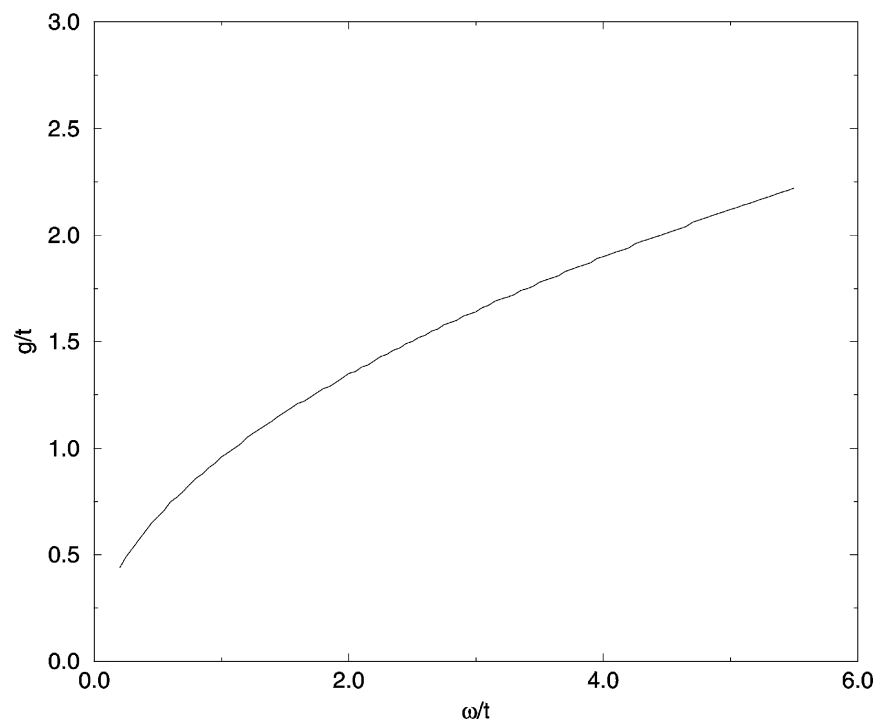

Fig. 5. The critical value of $g / t$ as a function of $\omega / t$.

\section{Acknowledgements}

Authors are grately indebted to V.S. Yarunin, E.A. Kochetov and B.R. Bulka for the valuable discussions. M.Y.Zh. is grateful to TÜBITAK (Scientific and Technical 
Research Council of Turkey) and Bilkent University for support and hospitality. The work is partialy supported by the Russian-Turkish Project "Phonon Anomalies". M.Y.Zh. is also grateful to Bielefeld University for support and hospitality.

\section{Appendix}

In this Appendix, we calculate the path integral $\int \mathrm{D} w \mathrm{D} \bar{w} \mathrm{e}^{\delta^{2} S_{-}}$which is the Gaussian integral with the kernel depending on the difference of the times $\tau-\sigma$.

The action $\left(S_{-}\right)_{0}$ at stationary trajectories shifts the energy scale as

$$
\begin{aligned}
\left(S_{-}\right)_{0} & =\left.\int_{0}^{\beta}\left[-\bar{w} \dot{w}-\omega \bar{w} w-\frac{\Omega}{2}\right]\right|_{w=\bar{w}=W_{0-} / 2} \mathrm{~d} \tau \\
& =-\beta \frac{\omega W_{0-}^{2}}{4}+\beta \sqrt{t^{2}+\left(\varepsilon+\frac{g}{\sqrt{2}} W_{0-}\right)^{2}} \operatorname{sign}\left(\varepsilon+\frac{g}{\sqrt{2}} W_{0-}\right) .
\end{aligned}
$$

Here we take into account that

$$
\begin{aligned}
\left.\Omega\right|_{0} & =-t\left(z_{0}+\bar{z}_{0}\right)-\mathrm{i}\left(\varepsilon+g \sqrt{2} W_{0-}\right)\left(z_{0}-\bar{z}_{0}\right) \\
& =-2 \sqrt{t^{2}+\left(\varepsilon+\frac{g}{\sqrt{2}} W_{0-}\right)^{2}} \operatorname{sign}\left(\varepsilon+\frac{g}{\sqrt{2}} W_{0-}\right) .
\end{aligned}
$$

The effective action has the form

$$
\begin{aligned}
\delta^{2} S_{-}= & \int_{0}^{\beta} \int_{0}^{\beta}\left\{\left[\frac{1}{2}(\dot{\bar{w}} w-\bar{w} \dot{w})-\omega \bar{w} w\right] \delta(\tau-\sigma)+\frac{1}{2} \operatorname{sign}\left(\varepsilon+\frac{g}{\sqrt{2}} W_{0-}\right)\right. \\
& \times \frac{g^{2} t^{2}}{2 E_{0}^{2} \sinh \beta E_{0}} \cosh 2 E_{0}\left(\frac{\beta}{2}-|\tau-\sigma|\right) \\
& \times(\bar{w}(\tau)+w(\tau))(\bar{w}(\sigma)+w(\sigma))\} \mathrm{d} \tau \mathrm{d} \sigma,
\end{aligned}
$$

where $E_{0}=\sqrt{t^{2}+\left(\varepsilon+(g / \sqrt{2}) W_{0+}\right)^{2}}$. The calculation of the functional determinant, which is defined by the second variations can be performed with Fourier representation for the paths $w, \bar{w}[14]$.

$$
\begin{aligned}
S_{-}= & \beta\left\{-\omega \bar{w}_{0} w_{0}+\frac{g^{2} t^{2}}{4 E_{0}^{3}}\left(w_{0} w_{0}+\bar{w}_{0} \bar{w}_{0}+2 \bar{w}_{0} w_{0}\right)\right\} \\
& +\beta \sum_{n=1}^{\infty}\left\{\mathrm{i} \omega_{n}\left(\bar{w}_{n} w_{n}-\bar{w}_{-n} w_{-n}\right)-\omega\left(\bar{w}_{n} w_{n}+\bar{w}_{-n} w_{-n}\right)\right. \\
& \left.+\frac{2 g^{2} t^{2}}{E_{0}\left(\omega_{n}^{2}+4 E_{0}^{2}\right)}\left(w_{n} w_{-n}+\bar{w}_{n} \bar{w}_{-n}+\bar{w}_{n} w_{n}+\bar{w}_{-n} w_{-n}\right)\right\} .
\end{aligned}
$$


The action (A.3) in Fourier representation can be represented as

$$
\begin{aligned}
S_{-}= & \beta\left\{-\omega \bar{w}_{0} w_{0}+\frac{g^{2} t^{2}}{4 E_{0}^{3}}\left(w_{0} w_{0}+\bar{w}_{0} \bar{w}_{0}+2 \bar{w}_{0} w_{0}\right)\right\}+\beta \sum_{n=1}^{\infty}\left(\bar{w}_{n} w_{-n}\right) \\
& \times\left(\begin{array}{cc}
\mathrm{i} \omega_{n}-\omega+K /\left(\omega_{n}^{2}+4 E_{0}^{2}\right) & K /\left(\omega_{n}^{2}+4 E_{0}^{2}\right) \\
K /\left(\omega_{n}^{2}+4 E_{0}^{2}\right) & -\mathrm{i} \omega_{n}-\omega+K /\left(\omega_{n}^{2}+4 E_{0}^{2}\right)
\end{array}\right)\left(\begin{array}{c}
w_{n} \\
\bar{w}_{-n}
\end{array}\right),
\end{aligned}
$$

where $K=2 g^{2} t^{2} / E_{0}, \omega_{n}=2 \pi n / \beta$. We calculate the ratio $Z_{w-}(g) / Z_{w-}(g=0)$ :

$$
\begin{aligned}
Z_{w-}(g) / Z_{w-}(g=0) \\
=\frac{\int \mathrm{d} w_{0} \mathrm{~d} \bar{w}_{0} \exp \left[-\left(\omega-K /\left(4 E_{0}^{2}\right)\right) \bar{w}_{0} w_{0}+\left((K / 2) /\left(4 E_{0}^{2}\right)\right)\left(w_{0} w_{0}+\bar{w}_{0} \bar{w}_{0}\right)\right]}{\int \mathrm{d} w_{0} \mathrm{~d} \bar{w}_{0} \exp \left[-\omega \bar{w}_{0} w_{0}\right]} \\
\quad \times \prod_{n=1}^{\infty} \frac{\omega_{n}^{2}+\left(\left(K / \omega_{n}^{2}+4 E_{0}^{2}\right)-\omega\right)^{2}-\left(K^{2} /\left(\omega_{n}^{2}+4 E_{0}^{2}\right)^{2}\right)}{\omega_{n}^{2}+\omega^{2}} \\
=\frac{\sinh \beta E_{0} \sinh (\beta \omega / 2)}{\sinh \left(\beta \omega_{1} / 2\right) \sinh \left(\beta \omega_{2} / 2\right)} .
\end{aligned}
$$

\section{References}

[1] A.S. Alexandrov, V.V. Kabanov, D.K. Ray, Phys. Rev. B 49 (1994) 9915.

[2] T. Hakioğlu, M.Ye. Zhuravlev, Phys. Rev. B 58 (1998) 3777.

[3] A. Kongeter, M. Wagner, J. Chem. Phys. 92 (1990) 4003.

[4] M.J. Rice, Solid State Commun. 31 (1979) 93.

[5] J. Ranninger, U. Thibblin, Phys. Rev. B 45 (1992) 7730.

[6] R. Fulton, M. Gouterman, J. Chem. Phys. 35 (1961) 1059.

[7] R. Pucci, V.S. Yarunin, M.Ye. Zhuravlev, J. Phys. A 31 (1998) 3185.

[8] M. Wagner, Unitary Transformations in Solid State Physics, Series on Modern Problems in Condensed Matter Sciences, Vol. 15, Elsevier, Amsterdam, 1986, 357 pp.

[9] M.J. Rice, V.M. Yartsev, C.S. Jacobsen, Phys. Rev. B 21 (1980) 3437.

[10] H. Kuratsuji, T. Suzuki, J. Math. Phys. 21 (1980) 472.

[11] A. Inomata, H. Kuratsuji, C.C. Gerry, Path Integrals and Coherent States of SU(2) and SU(1,1), World Scientific, Singapore, 1992.

[12] E.A. Kochetov, J. Math. Phys. 36 (1995) 4667.

[13] E.A. Kochetov, Phys. Rev. B 52 (1995) 4402.

[14] V.N. Popov, V.S. Yarunin, Collective Effects in Quantum Statistics of Radiation and Matter, Series on Mathematical Physics Studies, Vol. 9, Kluwer, Dordrecht, 1986.

[15] V.A. Ivanov, M.Ye. Zhuravlev, T. Hakioğlu, V.S. Yarunin, J. Phys. A 32 (1999) L361.

[16] V.V. Kabanov, O.Yu. Mashtakov, Phys. Rev. B 47 (1993) 6060.

[17] A.H. Romero, D.W. Brown, K. Lindenberg, Phys. Rev. B 60 (1999) 4618.

[18] M. Wagner, J. Phys. A 17 (1984) 2319. 